\title{
The Digital Engagement of Older People: Systematic Scoping Review Protocol
}

Abraham Sahilemichael Kebede ${ }^{1}$, MHS; Lise-Lotte Ozolins ${ }^{2}$, PhD; Hanna Holst ${ }^{2}$, PhD; Kathleen Galvin ${ }^{1}, \mathrm{PhD}$

${ }^{1}$ School of Health Sciences, University of Brighton, Brighton, United Kingdom

${ }^{2}$ Department of Health and Caring Sciences, Linnaeus University, Växjö, Sweden

\section{Corresponding Author:}

Abraham Sahilemichael Kebede, MHS

School of Health Sciences

University of Brighton

Village Way

Brighton, BN1 9PH

United Kingdom

Phone: 447502375585

Email: a.s.kebede@brighton.ac.uk

\section{Abstract}

Background: There is an ongoing negative narrative about aging that portrays older people as a socioeconomic burden on society. However, increased longevity and good health will allow older adults to contribute meaningfully to society and maximize their well-being. As such, a paradigm shift toward healthy and successful aging can be potentially facilitated by the growing digital technology use for mainstream (day-to-day activities) and assisted living (health and social care). Despite the rising digital engagement trend, digital inequality between the age groups persists.

Objective: The aims of this scoping review are to identify the extent and breadth of existing literature of older people's perspectives on digital engagement and summarize the barriers and facilitators for technological nonuse, initial adoption, and sustained digital technology engagement.

Methods: This review will be based on the Arksey and O'Malley framework for scoping reviews. The 6-stage framework includes: identifying research questions, identifying relevant studies, study selection, charting the data, summarizing and reporting the results, and a consultation exercise. Published literature will be searched on primary electronic databases such as the Association of Computing Machinery, Web of Science, MEDLINE, PsycINFO, CINAHL, and ScienceDirect. Common grey literature sources will complement the database search on the topic. A two-stage (title/abstract and full article) screening will be conducted to obtain eligible studies for final inclusion. A standardized data extraction tool will be used to extract variables such as the profile of the study population, technologies under investigation, stage of digital engagement, and the barriers and facilitators. Identified and eligible studies will be analyzed using a quantitative (ie, frequency analysis) and qualitative (ie, content analysis) approach suitable for comparing and evaluating literature to provide an evaluation of the current state of the older person's digital engagement. Inclusion will be based on the Joanna Briggs Institute-recommended participant, concept, and context framework. Articles on older people (65 years and older), on digital technology engagement, and from a global context will be included in our review.

Results: The results of this review are expected in July 2021.

Conclusions: The findings from this review will identify the extent and nature of empirical evidence on how older people digitally engage and the associated barriers and facilitators.

International Registered Report Identifier (IRRID): PRR1-10.2196/25616

(JMIR Res Protoc 2021;10(7):e25616) doi: $\underline{10.2196 / 25616}$

\section{KEYWORDS}

digital divide; digital engagement; digital inclusion; initial adoption; older people; older users; sustained engagement; technological nonuse; older adults 


\section{Introduction}

\section{Background}

Global demographic trends show that the worldwide age structure is rapidly changing more than ever before. The United Nations defines older people as those aged 65 years or older based on people's chronological age. Currently, there are over 703 million older people, and it is expected to reach 2.1 billion by the year $2050[1,2]$. Population projections have indicated Europe and North America have the fastest growing aging population, and by 2050 , the population percentage of older adults is expected to reach $34 \%$ in Europe and $28 \%$ in North America [3].

There is an ongoing negative narrative about aging that age-related changes, disability, and dependency among older people with poor and deteriorating health conditions imply an increased expenditure on health and its burden on the socioeconomic aspects of society [4]. Further, the COVID-19 pandemic has also underlined how older people are generally perceived and valued in our contemporary society [5-7]. This crisis exacerbated existing and deeply rooted inequalities such as underfinancing in the care sector and the chronic shortage of caregivers (both in the health and social sector) [8]. However, contrary to the negative narrative, increased longevity and good health allow older adults to meaningfully contribute socially and economically, and maximize their well-being late into life [9-11]. To facilitate healthy and successful aging, the fast-growing digital technology, with all its drawbacks, barriers, and challenges, offers a staggering promise and opportunity [12].

Despite substantial mixed and inconclusive findings, several studies and reviews have demonstrated the positive impact of digital technologies on different dimensions of an older person's life, including health, housing, services and transactions, mobility and transportation, access to information, communication and work, recreation, and self-fulfillment [13-15]. Moreover, digital technologies play a substantial role in improving older people's quality of life and independence [16-18]. However, a review reported an ambivalence toward digital technology due to negative effects such as a sense of privacy and personal security breaches. Whereas, personal safety during emergencies was reported as a positive effect of owning a mobile phone [18].

Over the past decades, digital technology use among older populations has grown exponentially both in the mainstream (day-to-day lives) and assisted care (health and social care) $[19,20]$. Changes in the workplace and the "digital by default" strategy for delivering public services are among contributing factors forcing older people to engage digitally [21]. Digital engagement in health promotion and social support through health information is also growing. However, the breadth and the extent of digital technology use among older people remains limited to communications such as sending or receiving emails, instant messaging, video calls (Skype), and making voice calls [14]. A perceived or actual lack of interest, skill gaps, and socioeconomic factors were mentioned as possible reasons for the limited use of digital technologies [14]. Besides, the age-related decline in vision, hearing, cognition, and dexterity also attribute to the limited use of digital technologies [22-24].

Comparatively, there is a discrepancy in digital involvement, access, and connectivity between the younger and older populations [16,24]. For instance, in the United Kingdom between 2014 and 2019, a significant proportion of the older population never connected digitally at all or had not used the internet over the past 3 months. The 2019 Office for National Statistics (ONS) survey showed $13.5 \%$ of older people aged 65 to 74 years old and $47 \%$ of those 75 years and older never used the internet [16]. A similar population-based study in 7 European countries reported only $12 \%$ internet use among older people (60 years and older), of whom $64 \%$ used it for health-related issues [25]. In the United States, smartphone ownership among older people 65 years and older is significantly lower in comparison to the national average $(81 \%$; ie, $59 \%$ of those between the ages of 65 and 74 years are smartphone owners, but it falls to $40 \%$ among those 75 years and older) [26].

To create a digitally inclusive and accessible world, the International Organization for Standardization recommends human-centered and accessible designs (ISO 9241-11:2018) [27]. Adaptation guidelines such as text font size, screen setting, contrast, and color adjustments are among the recommended standards. These modalities enable older people with physical disabilities to engage digitally [28]. However, technology designs are mostly driven by technology push rather than user demand pull factors. Additionally, the fast-evolving nature of digital technology makes it challenging for older people to catch up and sustain engagement with the adaptation guidelines.

\section{Digital Engagement Later in Life}

To thrive in the increasingly digitalized world, an acquaintance with technology is inadvertently becoming a mandatory way of life [21]. Despite the current assumption that older people are not using digital technologies, many studies have indicated that older people are competent and skilled digital technology users [29,30]. Still, there is a gap in evidence, with some key questions that require illumination:

1. What are the contributing factors to the digital inequality between the age groups?

2. How can we understand older people's digital technology use?

3. What constitutes the diversity of digital technology use?

The term "digital engagement or disengagement" has been widely used in marketing research with an aim toward promoting marketing strategies to end consumers [31-33]. Factors like brand factor, product factor, consumer factors, and content factors have been the main focus of these studies [34]. Though the factors are intertwined, this review will focus on studies that explore drivers of technological nonuse, initial adoption, and sustained digital engagement from older people's perspectives.

Overall, we propose to understand the current state of knowledge about older people's digital engagement through the stages of digital engagement (nonuse, initial adoption, and sustained engagement). This will facilitate an ongoing drive to reduce digital inequality and, in doing so, provide new understandings to promote the well-being of older people. It will also help 
identify potential alternatives for older people who remain nonusers of digital technology.

\section{Digital Engagement Dimensions}

To facilitate this review, operationalizing older people's digital engagement and disengagement is considered an important step in deciphering the continuum (Figure 1). This continuum with a three-stage approach involves technological nonuse, initial adoption or acceptance, and sustained digital engagement. This categorization will enrich the evidence mapping and the identification of barriers and facilitators for each dimension (initial adoption, sustained engagement, and technology nonuse). The description for each digital engagement dimension is provided in the following sections.

Figure 1. Older people's digital engagement dimensions and stages later in life.

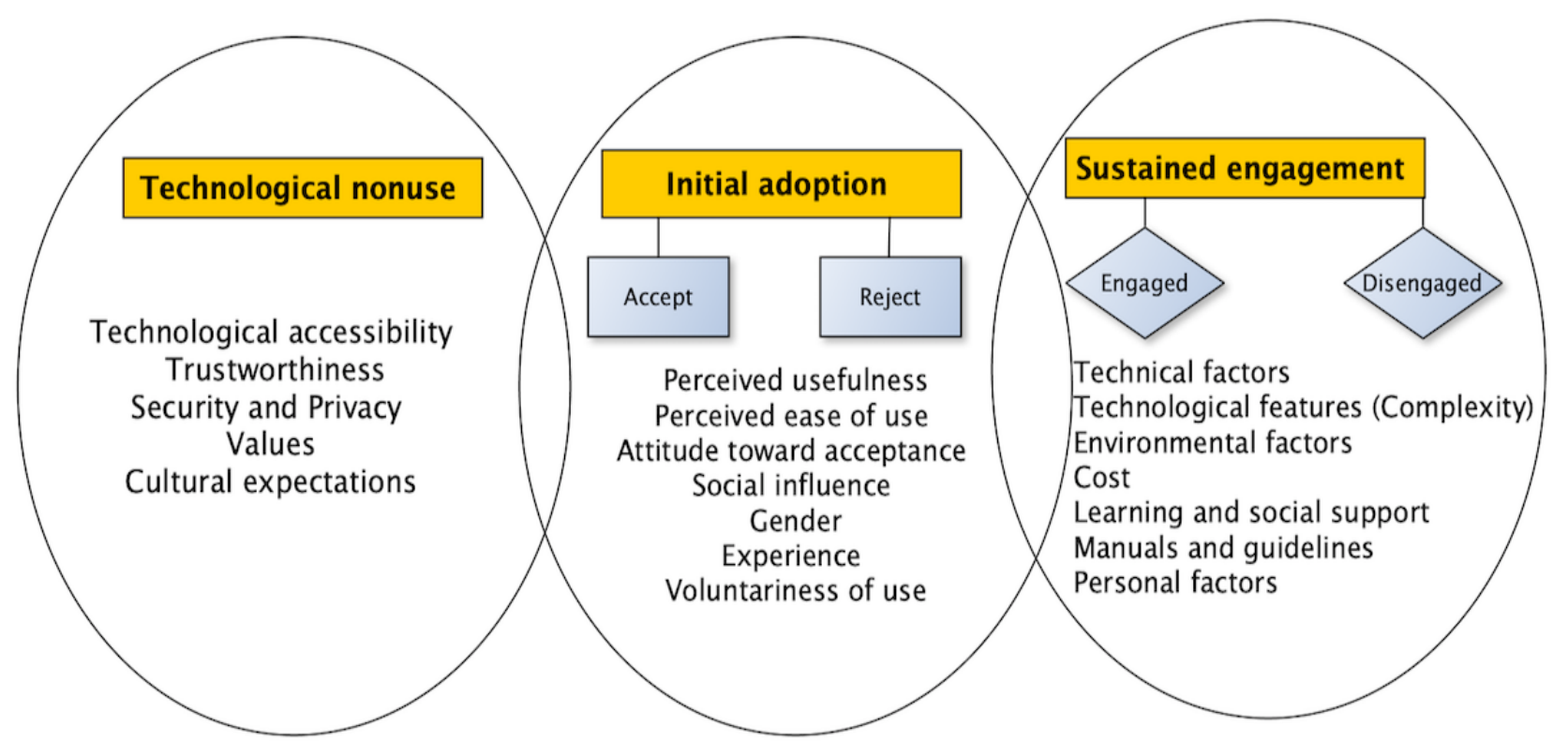

Older people digital engagment stages

\section{Stage I: Digital Technology Nonuse}

Technological nonuse is not absolute as the term may suggest and goes beyond the absence of technology [35]. It is also a mistake to assume a person has not used a single digital technology, as use or nonuse is a constant negotiation and renegotiation to engage or disengage with technology. This also includes older people who access digital technology through their existing social support system (family and friends). To understand the possible factors affecting older people's engagement and disengagement, efforts to investigate the technological nonuse should be encouraged $[35,36]$. This will pave the way to understanding the bigger picture of digital exclusion among older people.

Governments across Europe (eg, the United Kingdom, Sweden, and Spain) have shown commitments to provide digital technologies through a framework (eg, universal service obligations for broadband) and accessible internet to citizens [37-39]. However, evidence has indicated that technological nonuse, later in life digital disengagement, and lower use rates are the main features of digital inequality among the older population $[21,40]$. The nonuse might involve technology, a service, an application, a platform, a communication medium, a set of practices, or some combination thereof. For example, the 2019 ONS survey in the United Kingdom showed $13.5 \%$ of older people aged 65 to 74 years and $47 \%$ of those 75 years and older never used the internet [16].

The drivers of technological nonuse are not only limited to sociodemographic and economic characteristics, but also the absence of tailored instructions and guidance, a lack of knowledge and confidence, and health-related barriers and costs [41]. According to Knowles and Hanson [17], accessibility and trustworthiness of the digital technologies, values, and religious and cultural expectations are salient determinants for older people's technological nonuse. Moreover, complexity, security, and privacy issues also contribute to the technological nonuse among this age group.

\section{Stage II: Initial Adoption}

Studies dealing with user (older person) decisions to accept or reject digital technology and the drivers that influence the user decision will inform this stage. This will answer questions such as, "What influences users' decision to use a particular digital technology?"

A considerable range of models and theories such as the Theory of Reasoned Action (TRA) [42], the Technological Acceptance Model (TAM) [43], the Unified Theory of Acceptance and Use of Technology (UTAUT) [44], the Diffusion of Innovations Theory [45], and Igbaria's model [46] have been developed to facilitate an understanding of the drivers toward the 
favorableness and the unfavorableness of technology initial adoption [47]. The TAM [43,48] and UTAUT [44], a derivative of TRA, are among the prevailing theories. The TAM developed antecedent factors such as perceived usefulness, perceived ease of use, and attitude toward technological acceptance. Whereas, UTAUT, which is the extension of TAM, further developed the model by adding social influence and other moderating factors such as gender, age, experience, and voluntariness of use [44]. This review will scope studies that address these factors with age (65 years and older) as an important moderating factor [44]. Furthermore, this review will include qualitative accounts from older people's perspectives, unlike the TAM and UTAUT models, which are widely used to quantify acceptance [49].

\section{Stage III: Sustained Digital Engagement}

People who actively used technology start to disengage due to age or the generational effects of aging [30]. According to Damodaran et al [50], sustained digital engagement is affected by the complexity and fast-changing nature of digital technology. Additionally, user's low awareness about the availability of design adaptation modalities such as font size, color, and screen determined its sustained use. The manuals and guidelines on this design adaptation, which enhance older people's capacity to adapt to technologies, are frequently inaccessible and outdated. Learning and support from existing social support such as family play a crucial role at this stage [50]. A similar study reported that sustained mobile technology use among older people was influenced by personal factors (physical, cognitive, and mental changes), environmental factors (financial costs, social influence, and learning to use technology), and technical factors (complexity and usability, absence of feedback, and design challenge) [29].

Sustained use is vital in understanding the digital divide among different socioeconomic groups [50]. However, studies suggested that it is one of the underresearched areas of digital engagement. A growing body of evidence has focused on understanding the early adoption, with the assumption that once people subscribe to the technology, they will keep using it. However, there is evidence that there will be a digital engagement negotiation and renegotiation between use and nonuse and vice versa [21]. Therefore, this review will include studies focused on factors that prevent or promote sustained use among older people.

\section{Scoping Review Rationale}

There is a growing body of literature that often gives glowing reviews on the positive effect of digital technology engagement among older people. However, there is a gap in comprehensive reviews of evidence understanding the complexities of the barriers and facilitators of older people's digital engagement. This review will summarize the current state of knowledge concerning older people's perspectives on digital engagement and disengagement from technological nonuse, initial adoption, and sustained use. In addition, the varieties of technologies used or being used in social and health care for older people will be identified.

Studies have shown that the use of digital technology will have a great impact on different dimensions of older people's lives, for example, quality of life [18], decision making [29], and mobility and social connectedness [14]. However, there are no reviews of existing studies that summarized the state of knowledge from older people's perspectives, specifically the drivers of engagement and disengagement from technological nonuse to initial adoption and sustained use. This scoping review aims to provide a base for a more comprehensive understanding of digital engagement among older people. The findings will inform older people, designers, developers, and decision makers about practical implications. In addition, this review will set an agenda for future research and further in-depth understanding of older people's digital engagement.

The findings from this review will inform the extent of evidence on older people's digital engagement, inform the extent and the breadth of the knowledge about barriers and facilitators of older people's digital engagement, and delineate the scope of what we already know. Further, these findings will indicate the gaps in the ongoing research of the issue.

\section{The Rationale in Light of the COVID-19 Pandemic}

As of November 2020, there have been over 50 million COVID-19 cases and over 1 million deaths worldwide. Governments worldwide have implemented different levels of public health infrastructures such as lockdowns, social distancing, testing, contact tracing, and isolation measures [51]. As a result, digital technology use as a modality for coping with the crisis and socioeconomic continuity has substantially increased. For example, people are now using technology to work from home, to speak to their families and loved ones, and to source entertainment and information [52,53]. In addition, contact tracing apps were implemented in European countries, China, Singapore, and the United Kingdom [54,55]. Despite the unanticipated nature of the crisis and the higher vulnerability associated with age, the existing digital technology inequality among the age groups could imply low use or uptake of such services for the well-being of an older person, exacerbating the existing inequality [56]. In this new configuration of societal roles and innovative ways to tackle the transmission and stop the pandemic, future understanding of older people's digital engagement will shed light on existing efforts to make technologies equitable.

\section{Methods}

\section{Overview}

The methodology for this scoping review is informed mainly by the Arksey and O'Malley framework for scoping reviews and will examine the extent, range, and breadth of evidence for the drivers of digital engagement among older people [57]. Additional recent methodological development on scoping reviews by Levac et al [58] and Tricco et al [59] (ie, PRISMA-ScR [Preferred Reporting Items for Systematic Reviews and Meta-Analyses Extension for Scoping Reviews] Checklist) will be incorporated in the main framework. The framework has 6 steps described in the following sections. 


\section{Stage 1: Identifying Research Questions}

Identifying relevant and broader research questions is the first step in the process of a scoping review. Our review questions are as follows:

1. What is known from the existing literature about the perspectives of older people on digital technology engagement?

2. What digital technologies have been used in the health and social care of older people?

\section{Stage 2: Identifying Relevant Studies}

A comprehensive search of identified electronic databases will be conducted to locate relevant studies. Our search will include primary databases such as the Association for Computing Machinery Digital Library; Library, Information Science, and Technology Abstracts; MEDLINE; PsycINFO; CINAHL; and ScienceDirect. The search will be complemented by interdisciplinary (Web of Science, EBSCO, and Scopus) and secondary databases (Cochrane library and Joanna Briggs Institute [JBI] reviews). In addition, common grey literature sources from key journals (JMIR, the Journal of Gerontology, and the Journal of Gerontechnology) and Google Scholar will be included. Additional manual searches of peer-reviewed and grey source literature on the current COVID-19 crisis and the role of digital technology engagement among older people, published from December 2019 onward, will be included to support the review rationale.

Taking into consideration the research question and the JBI recommended population, concept, and context (PCC) approach, keywords and their synonyms, plurals, spellings, and acronyms will be used to develop a comprehensive search strategy as follows.

1. The population of this study is limited to studies conducted among older people 65 years and older. Terms such as “older person," OR “older people," OR “elderly," OR "geriatric," OR “old," OR “frail," OR “older user” will be used to form the population.

2. The concept will include studies dealing with digital engagement. Terms such as "digital," OR "digital technology," OR “digital engagement," OR "digital technology engagement," OR "technology" will form the concept.

3. There will be no restriction by context in terms of the geography of the studies.

All identified literature from our broad search strategy will be exported to the EndNote library manager and Evidence for Policy and Practice Information (EPPI) Reviewer 4 for the two-stage screening (title/abstract and full article).

\section{Stage 3: Study Selection}

Inclusion and exclusion criteria to select studies will be generated based on the scope of the inquiry. Accordingly, we will use the iterative search strategy as we go back and forth to refine the search strategy and study selection.

\section{Inclusion Criteria}

Peer-reviewed articles will be the primary target, but also, grey literature sources with important insights into the scope will also be included to enrich the review. The inclusion criteria will be in line with the PCC of the studies described as follows:

1. Participants/Population: In this scoping review, we will include only literature that focused on digital technology among older people aged 65 years and older. Technological research with different age groups or a cross-age categorization will be excluded.

2. Concept/Condition: The primary concept in this study will be digital engagement. Studies that investigated digital engagement among older people and the determinants for technological nonuse, initial adoption, and sustained digital use will be included. In addition, studies that investigated different aspects of older people's digital technology engagement, digital inclusion and divide, and other intersection features between old age and digital technology will be included.

3. Context: The context of this study will be global.

\section{Study Identification}

The study selection will involve two stages of screening. EPPI Reviewer software version 4 (from Evidence for Policy and Practice Information and Co-ordinating Centre) will be used to facilitate the screening process.

1. Title and abstract screening will be performed according to the inclusion and exclusion criteria.

2. Articles qualified by the title and abstract screening will be further considered for full article appraisal. Full articles will be accessed through the University of Brighton library, interuniversity library resources, and contacting the authors. The search results, screening process, and reasons for exclusion will be presented using the PRISMA-ScR flow diagram.

\section{Stage 4: Charting the Data}

Important variables from studies found to be eligible for final inclusion in the scoping review will be extracted using a customized data extraction tool. The extracted variables will inform the scope and the breadth of the existing literature on older people's digital engagement (Textbox 1). Variables such as study design, source of data, study size, study setting, study population, digital technology used, stage of digital engagement under study, and the barriers and facilitators of digital engagement among older people will be extracted. 
Textbox 1. Variables to be extracted by review questions.

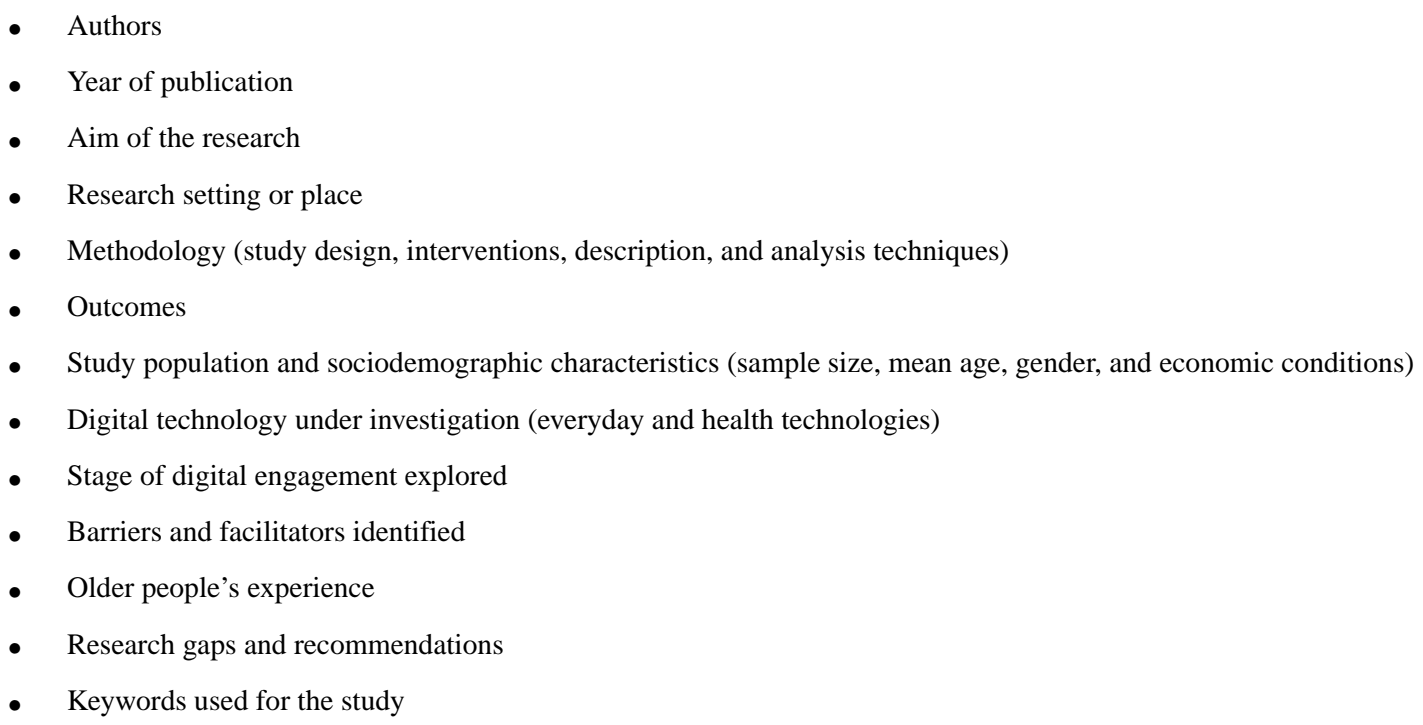

\section{Stage 5: Collating, Summarizing, and Reporting the Results}

An extension of the PRISMA-ScR flow diagram and guideline for reporting scoping reviews will be used to describe and collate the results of the final review $[58,59]$. The scoping will involve quantitative analysis (ie, frequency analysis), numerical description and common characterization of the studies by a study setting (geography or distribution), type of the study designs, the mean age of the study participants, and other features. Finally, the qualitative analysis will be conducted using the content analysis technique. Conceptual categories and definitions will be formed to inform the meanings, barriers, facilitators, and experiences of older people related to digital technology engagement. These categories will be used to generate themes. Levac et al [58] recommended qualitative content analysis to facilitate the summary and make sense of the extracted variables. This relational conceptual analysis will help explore relationships between the concepts extracted from the articles in the field. Charting of important variables and a narrative description of the findings will be presented in the review report.

\section{Stage 6: Consultation Exercise}

We will conduct a consultation based on the identified preliminary literature findings on the topic of interest with identified stakeholders including advocacy groups, older people, academicians, digital developers, practitioners, and other early-stage researchers. This consultation exercise will be done after the preliminary electronic search on the common databases. The findings from the consultation exercise will inform our revision of the research question and refine the search strategy. The findings from the consultation exercise will be thematically presented.

\section{Dissemination and Ethical Requirements}

We will comment on the ethical approval status of the included studies. However, for this review, ethical approval is not required since it uses publicly available sources. The key finding from this scoping review will be made available online and will be disseminated to key stakeholders.

\section{Results}

We have conducted a preliminary search of the primary databases. We expect the final database search of this review to be completed in May 2021. We envisage disseminating the findings from this systematic scoping review in a scientific peer-reviewed journal.

\section{Discussion}

We conceptualized older people's digital engagement in a three-stage continuum from nonuse and initial adoption to sustained engagement. The findings from this review will identify the extent and nature of empirical evidence on how older people digitally engage and the associated barriers and facilitators at each stage of the continuum.

\section{Acknowledgments}

This project has received funding from the European Union's Horizon 2020 research and innovation program under grant agreement No. 813928.

\section{Authors' Contributions}

ASK developed the research questions and study methods and designed this protocol. KG refined the research questions and helped develop the research and study methods. KG, LLO, and HH reviewed the methodology and contributed meaningfully to the drafting and editing of the manuscript. The final manuscript version was read and approved by all authors. 


\section{Conflicts of Interest}

None declared.

\section{References}

1. United Nations, Department of Economic and Social Affairs, Population Division. World Population Prospects 2019: Highlights. Division, P. Geneva; 2019. URL: https://population.un.org/wpp/ [accessed 2020-06-14]

2. United Nations, Department of Economic and Social Affairs, Population Division. World Population Ageing. 2017. URL: https://www.un.org/en/development/desa/population/publications/pdf/ageing/WPA2017 Highlights.pdf [accessed 2020-10-21]

3. European Commission. The 2018 Ageing Report; Economic \& Budgetary Projections for the 28 EU Memeber States(2016-2070). Luxembourg: Office of the European Union; 2018. URL: https://ec.europa.eu/info/publications/ economy-finance/2018-ageing-report-economic-and-budgetary-projections-eu-member-states-2016-2070 en [accessed 2021-03-20]

4. Bernard M. Promoting Health in Old Age: Critical Issues in Self Health Care. Philadelphia, PA: Open University Press; 2000 .

5. Cecula P, Behan SD, Maruthappu M. COVID-19: Challenges and opportunities in the care sector. EClinicalMedicine 2020 Jun;23:100390 [FREE Full text] [doi: 10.1016/j.eclinm.2020.100390] [Medline: $\underline{\text { 32395708] }}$

6. Fraser S, Lagacé M, Bongué B, Ndeye N, Guyot J, Bechard L, CCNA Social InclusionStigma Working Group, et al. Ageism and COVID-19: what does our society's response say about us? Age Ageing 2020 Aug 24;49(5):692-695 [FREE Full text] [doi: 10.1093/ageing/afaa097] [Medline: $\underline{32377666]}$

7. Flett GL, Heisel MJ. Aging and feeling valued versus expendable during the COVID-19 pandemic and beyond: a review and commentary of why mattering is fundamental to the health and well-being of older adults. Int J Ment Health Addict 2020 Jun 15:1-27 [FREE Full text] [doi: 10.1007/s11469-020-00339-4] [Medline: 32837430]

8. The Guardian. The Guardian view on social care and Covid-19: the neglected frontline. URL: https://www.theguardian.com/ world/2020/mar/25/the-guardian-view-on-social-care-and-covid-19-the-neglected-frontline [accessed 2020-11-02]

9. Bloom DE, Canning D, Fink G. Implications of population ageing for economic growth. Oxford Review of Economic Policy 2011 May 13;26(4):583-612. [doi: 10.1093/oxrep/grq038]

10. King M, Lipsky MS. Clinical implications of aging. Dis Mon 2015 Nov;61(11):467-474. [doi: 10.1016/j.disamonth.2015.09.006] [Medline: 26497929]

11. Murman D. The impact of age on cognition. Semin Hear 2015 Aug 9;36(3):111-121 [FREE Full text] [doi: 10.1055/s-0035-1555115] [Medline: 27516712]

12. Soumitra D, Thiery G, Bruno L. The Global Information Technology Report 2015; ICTs for Inclusive Growth. Geneva: World Economic Forum; 2015. URL: http://www3.weforum.org/docs/WEF Global IT Report 2015.pdf [accessed 2020-09-12]

13. L. Fozard, Jan Rietsema, Herman Bou J. Gerontechnology: creating enabling environments for the challenges and opportunities of aging. Educational Gerontology 2000 Jun;26(4):331-344. [doi: 10.1080/036012700407820]

14. Davidson S. Digital Inclusion Evidence Review, AgeUK. 2018. URL: https://www.ageuk.org.uk/globalassets/age-uk/ documents/reports-and-publications/age uk digital inclusion evidence review 2018.pdf [accessed 2020-11-05]

15. Chopik WJ. The benefits of social technology use among older adults are mediated by reduced loneliness. Cyberpsychol Behav Soc Netw 2016 Sep;19(9):551-556 [FREE Full text] [doi: 10.1089/cyber.2016.0151] [Medline: 27541746]

16. Office of National Statistics. Internet Users 2019. 2020. URL: https://www.ons.gov.uk/businessindustryandtrade/ itandinternetindustry/datasets/internetusers [accessed 2020-10-18]

17. Knowles B, Hanson VL. The wisdom of older technology (non)users. Commun. ACM 2018 Feb 21;61(3):72-77. [doi: $10.1145 / 3179995]$

18. Damant J, Knapp M, Freddolino P, Lombard D. Effects of digital engagement on the quality of life of older people. Health Soc Care Community 2017 Nov 25;25(6):1679-1703. [doi: 10.1111/hsc.12335] [Medline: 26919220]

19. Ageing in place. Technology In Our Life Today And How It Has Changed. 2019. URL: https://www.aginginplace.org/ technology-in-our-life-today-and-how-it-has-changed/ [accessed 2020-06-22]

20. Steinhubl SR, Wolff-Hughes DL, Nilsen W, Iturriaga E, Califf RM. Digital clinical trials: creating a vision for the future. NPJ Digit Med 2019;2:126 [FREE Full text] [doi: 10.1038/s41746-019-0203-0] [Medline: $\underline{31872066}$ ]

21. Olphert W, Damodaran L. Older people and digital disengagement: a fourth digital divide? Gerontology 2013;59(6):564-570. [doi: 10.1159/000353630] [Medline: 23969758]

22. Vaportzis E, Clausen MG, Gow AJ. Older adults perceptions of technology and barriers to interacting with tablet computers: a focus group study. Front Psychol 2017 Oct 04;8:1687 [FREE Full text] [doi: 10.3389/fpsyg.2017.01687] [Medline: 29071004]

23. Blaschke CM, Freddolino PP, Mullen EE. Ageing and technology: a review of the research literature. British Journal of Social Work 2009 Mar 20;39(4):641-656. [doi: 10.1093/bjsw/bcp025] 
24. Francis J, Christopher B, Travis K, Sheila RC. Aging in the digital age: conceptualizing technology adoption and digital inequalities. Ageing and Digital Technology: Designing and Evaluating Emerging Technologies for Older Adults 2019; Springer:35-49. [doi: 10.1007/978-981-13-3693-5 3]

25. Wangberg SC, Andreassen HK, Prokosch H, Santana SMV, Sørensen T, Chronaki CE. Relations between Internet use, socio-economic status (SES), social support and subjective health. Health Promot Int 2008 Mar 01;23(1):70-77. [doi: 10.1093/heapro/dam039] [Medline: 18083686]

26. Pew Research Center. Mobile Technology and Home Broadband 2019. 2019. URL: https://www.pewresearch.org/internet/ 2019/06/13/mobile-technology-and-home-broadband-2019/ [accessed 2021-03-10]

27. British Standards Institution. BS EN ISO 9241-11:1998. Ergonomic requirements for office work with visual display terminals (VDTs). Guidance on usability. 2018. URL: https://landingpage.bsigroup.com/LandingPage/ Standard?UPI=000000000030005417 [accessed 2020-09-24]

28. Rodeschini G. Gerotechnology: a new kind of care for aging? An analysis of the relationship between older people and technology. Nurs Health Sci 2011 Dec;13(4):521-528. [doi: 10.1111/j.1442-2018.2011.00634.x] [Medline: 21929580]

29. Kuerbis A, Mulliken A, Muench F, A. Moore A, Gardner D. Older adults and mobile technology: factors that enhance and inhibit utilization in the context of behavioral health. Ment Health Addict Res 2017;2(2):1-11. [doi: 10.15761/mhar.1000136]

30. Niemelä - Nyrhinen J. Baby boom consumers and technology: shooting down stereotypes. Journal of Consumer Marketing 2007 Aug 07;24(5):305-312. [doi: 10.1108/07363760710773120]

31. Calder BJ, Malthouse EC, Maslowska E. Brand marketing, big data and social innovation as future research directions for engagement. Journal of Marketing Management 2016 Apr 04;32(5-6):579-585. [doi: 10.1080/0267257x.2016.1144326]

32. Brodie RJ, Hollebeek LD, Jurić B, Ilić A. Customer engagement. Journal of Service Research 2011 Jul 06;14(3):252-271. [doi: $10.1177 / 1094670511411703$ ]

33. Bowden J. Customer engagement: a framework for assessing customer-brand relationships: the case of the restaurant industry. Journal of Hospitality Marketing \& Management 2009 Jul 10;18(6):574-596. [doi: 10.1080/19368620903024983]

34. Barger V, Peltier JW, Schultz DE. Social media and consumer engagement: a review and research agenda. JRIM 2016 Oct 10;10(4):268-287. [doi: 10.1108/jrim-06-2016-0065]

35. Baumer EPS, Burrell J, Ames MG, Brubaker JR, Dourish P. On the importance and implications of studying technology non-use. interactions 2015 Feb 25;22(2):52-56. [doi: 10.1145/2723667]

36. Christine S, Paul D. Beyond the user: use and non-use in HCI. New York, NY: Association for Computing Machinery; 2009 Presented at: The 21st Annual Conference of the Australian Computer-Human Interaction Special Interest Group; November 3-27, 2009; Melbourne, Australia p. 9-16. [doi: 10.1145/1738826.1738829]

37. Hutton G. The universal service obligation (USO) for broadband 2022, House of Commons Library. London: House of Commons Library; 2020 Oct 02. URL: https://commonslibrary.parliament.uk/research-briefings/cbp-8146/ [accessed 2020-12-18]

38. Telecompaper. Swedish govt wants 'universal' gigabit service by 2025. 2016. URL: https://www.telecompaper.com/news/ swedish-govt-wants-universal-gigabit-service-by-2025--1176709 [accessed 2020-10-19]

39. TeleGeography. Spain to add 1Mbps minimum speed to universal service obligation from 2011. 2009. URL: $\underline{\text { https://www. }}$ commsupdate.com/articles/2009/12/04/spain-to-add-1mbps-minimum-speed-to-universal-service-obligation-from-2011/ [accessed 2020-10-22]

40. Ofcom. Statement: Delivering the Broadband Universal Service. 2019. URL: https://www.ofcom.org.uk/ consultations-and-statements/category-1/delivering-broadband-universal-service [accessed 2020-10-22]

41. Reisdorf BC, Groselj D. Internet (non-)use types and motivational access: Implications for digital inequalities research. New Media \& Society 2015 Dec 27;19(8):1157-1176. [doi: 10.1177/1461444815621539]

42. Fishbein M, Ajzen I. Belief, Attitude, Intention, and Behavior: An Introduction to Theory and Research. MA: Addison-Wesley; 1975.

43. Davis FD. Perceived usefulness, perceived ease of use, and user acceptance of information technology. MIS Quarterly 1989 Sep;13(3):319. [doi: 10.2307/249008]

44. Venkatesh V, Morris MG, Davis GB, Davis FD. User acceptance of information technology: toward a unified view. MIS Quarterly 2003;27(3):425. [doi: 10.2307/30036540]

45. Rogers EM. Diffusion of Innovations. New York: The Free Press; 1995.

46. Igbaria M, Parasuraman S, Baroudi JJ. A motivational model of microcomputer usage. Journal of Management Information Systems 2015 Dec 11;13(1):127-143. [doi: 10.1080/07421222.1996.11518115]

47. Taherdoost H. A review of technology acceptance and adoption models and theories. Procedia Manufacturing 2018;22:960-967. [doi: 10.1016/j.promfg.2018.03.137]

48. Chung JE, Park N, Wang H, Fulk J, McLaughlin M. Age differences in perceptions of online community participation among non-users: An extension of the Technology Acceptance Model. Computers in Human Behavior 2010 Nov;26(6):1674-1684. [doi: 10.1016/j.chb.2010.06.016]

49. Venkatesh V, Davis FD. A model of the antecedents of perceived ease of use: development and test. Decision Sciences 1996 Sep;27(3):451-481. [doi: 10.1111/j.1540-5915.1996.tb01822.x] 
50. Damodaran L, Olphert C, Sandhu J. Falling off the bandwagon? Exploring the challenges to sustained digital engagement by older people. Gerontology 2014;60(2):163-173. [doi: 10.1159/000357431] [Medline: 24356488]

51. Anderson RM, Heesterbeek H, Klinkenberg D, Hollingsworth TD. How will country-based mitigation measures influence the course of the COVID-19 epidemic? The Lancet 2020 Mar;395(10228):931-934. [doi: 10.1016/s0140-6736(20)30567-5]

52. Chen X, Zhou X, Li H, Li J, Jiang H. The value of WeChat as a source of information on the COVID-19 in China. [Preprint]. Bull World Health Organ 2020 Mar 30:2020. [doi: 10.2471/blt.20.256255]

53. Brooks SK, Webster RK, Smith LE, Woodland L, Wessely S, Greenberg N, et al. The psychological impact of quarantine and how to reduce it: rapid review of the evidence. The Lancet 2020 Mar;395(10227):912-920. [doi: 10.1016/s0140-6736(20)30460-8]

54. eHealth Network. Mobile applications to support contact tracing in the EU's fight against COVID-19. Belgium; 2020. URL: https://ec.europa.eu/health/sites/default/files/ehealth/docs/covid-19 apps en.pdf [accessed 2020-09-18]

55. Freshfields Bruckhaus Deringer. Contact tracing apps in China, Hong Kong, Singapore, Japan, and South Korea. 2020 Apr 24. URL: https://digital.freshfields.com/post/102g5my/ contact-tracing-apps-in-china-hong-kong-singapore-japan-and-south-korea [accessed 2020-09-17]

56. Souter D. Inside the Digital Society: Inequality, the internet and the corona crisis.: Association for progressive communication London; 2020. URL: https://www.apc.org/en/blog/inside-digital-society-inequality-internet-and-corona-crisis [accessed 2020-09-22]

57. Arksey H, O'Malley L. Scoping studies: towards a methodological framework. International Journal of Social Research Methodology 2005 Feb;8(1):19-32. [doi: 10.1080/1364557032000119616]

58. Levac D, Colquhoun H, O'Brien KK. Scoping studies: advancing the methodology. Implement Sci 2010 Sep 20;5(1):69 [FREE Full text] [doi: 10.1186/1748-5908-5-69] [Medline: 20854677]

59. Tricco AC, Lillie E, Zarin W, O'Brien KK, Colquhoun H, Levac D, et al. PRISMA Extension for Scoping Reviews (PRISMA-ScR): checklist and explanation. Ann Intern Med 2018 Oct 02;169(7):467-473 [FREE Full text] [doi: 10.7326/M18-0850] [Medline: 30178033]

\author{
Abbreviations \\ EPPI: Evidence for Policy and Practice Information \\ JBI: Joanna Briggs Institute \\ ONS: Office for National Statistics \\ PCC: population, concept, and context \\ PRISMA-ScR: Preferred Reporting Items for Systematic Reviews and Meta-Analyses Extension for Scoping \\ Reviews \\ TAM: Technological Acceptance Model \\ TRA: Theory of Reasoned Action \\ UTAUT: Unified Theory of Acceptance and Use of Technology
}

Edited by G Eysenbach; submitted 09.11.20; peer-reviewed by K Colorafi, E Brainin, S Pit; comments to author 08.02.21; revised
version received 24.03.21; accepted 29.04.21; published 05.07.21
Please cite as:
Kebede AS, Ozolins LL, Holst H, Galvin K
The Digital Engagement of Older People: Systematic Scoping Review Protocol
JMIR Res Protoc 2021;10(7):e25616
URL: $\underline{\text { https://www.researchprotocols.org/2021/7/e25616 }}$
doi: $10.2196 / 25616$
PMID:

(C)Abraham Sahilemichael Kebede, Lise-Lotte Ozolins, Hanna Holst, Kathleen Galvin. Originally published in JMIR Research Protocols (https://www.researchprotocols.org), 05.07.2021. This is an open-access article distributed under the terms of the Creative Commons Attribution License (https://creativecommons.org/licenses/by/4.0/), which permits unrestricted use, distribution, and reproduction in any medium, provided the original work, first published in JMIR Research Protocols, is properly cited. The complete bibliographic information, a link to the original publication on https:/www.researchprotocols.org, as well as this copyright and license information must be included. 